\title{
Squarticles as the nanoantidotes to sequester the overdosed antidepressant for detoxification
}

\author{
This article was published in the following Dove Press journal: \\ International Journal of Nanomedicine \\ I November 2017 \\ Number of times this article has been viewed
}

\author{
Chun-Han Chen, ${ }^{1,2, *}$ Tse- \\ Hung Huang, ${ }^{3-5, *}$ Ahmed $\mathrm{O}$ \\ Elzoghby, ${ }^{6,7}$ Pei-Wen Wang, ${ }^{8}$ \\ Chia-Wen Chang, ${ }^{9}$ Jia-You \\ Fang ${ }^{9-12}$
}

'Division of General Surgery, Department of Surgery, Chang Gung Memorial Hospital, Chiayi, ${ }^{2} \mathrm{Graduate}$ Institute of Clinical Medical Sciences, College of Medicine, Chang Gung University, Kweishan, Taoyuan, ${ }^{3}$ Department of Traditional Chinese Medicine, Chang Gung Memorial Hospital, Keelung, ${ }^{4} \mathrm{School}$ of Traditional Chinese Medicine, Chang Gung University, Taoyuan, ${ }^{5} \mathrm{~S}$ chool of Nursing, National Taipei University of Nursing and Health Sciences, Taipei, Taiwan; ${ }^{6}$ Cancer Nanotechnology Research Laboratory (CNRL), ${ }^{7}$ Department of Industrial Pharmacy, Faculty of Pharmacy, Alexandria University, Alexandria, Egypt; ${ }^{8}$ Department of Medical Research, China Medical University Hospital, China Medical University, Taichung, ${ }^{9}$ Pharmaceutics Laboratory, Graduate Institute of Natural Products,

${ }^{10} \mathrm{Chinese}$ Herbal Medicine Research Team, Healthy Aging Research Center, Chang Gung University, "'Department of Anesthesiology, Chang Gung Memorial Hospital, ${ }^{12}$ Research Center for Industry of Human Ecology and Research Center for Chinese Herbal Medicine, Chang Gung University of Science and Technology, Kweishan, Taoyuan, Taiwan

*These authors contributed equally to this work

Correspondence: jia-You Fang Pharmaceutics Laboratory, Graduate Institute of Natural Products, Chang Gung University, 259 Wen-Hwa Ist Road, Kweishan, Taoyuan 333, Taiwan Tel +88632118800

Fax +88632118236

Email fajy@mail.cgu.edu.tw

\begin{abstract}
The increasing death rate caused by drug overdose points to an urgent demand for the development of novel detoxification therapy. In an attempt to detoxify tricyclic antidepressant overdose, we prepared a lipid nanoemulsion, called squarticles, as the nanoantidote. Squalene was the major lipid matrix of the squarticles. Here, we present the animal study to investigate both the pharmacokinetic and pharmacodynamic effects of squarticles on amitriptyline intoxication. The anionic and cationic squarticles had average diameters of 97 and $122 \mathrm{~nm}$, respectively. Through the entrapment study, squarticles could intercept $40 \%-50 \%$ of the amitriptyline during $2 \mathrm{~h}$ with low leakage after loading into the nanoparticles. The results of isothermal titration calorimetry demonstrated greater interaction of amitriptyline with the surface of anionic squarticles $\left(K_{\mathrm{a}}=28,700\right)$ than with cationic ones $\left(K_{\mathrm{a}}=5,010\right)$. Real-time imaging showed that intravenous administration of anionic squarticles resulted in a prolonged retention in the circulation. In a rat model of amitriptyline poisoning, anionic squarticles increased the plasma drug concentration by 2.5 -fold. The drug uptake in the highly perfused organs was diminished after squarticle infusion, indicating the lipid sink effect of bringing the entrapped overdosed drug in the tissues back into circulation. In addition, the anionic nanosystems restored the mean arterial pressure to near normal after amitriptyline injection. The survival rate of overdosed amitriptyline increased from $25 \%$ to $75 \%$ by treatment with squarticles. Our results show that the adverse effects of amitriptyline intoxication could be mitigated by administering anionic squarticles. This lipid nanoemulsion is a potent antidote to extract amitriptyline and eliminate it.
\end{abstract}

Keywords: squarticles, squalene, amitriptyline, overdose, antidote, pharmacokinetics

\section{Introduction}

Intentional drug poisoning is a common reason of Critical Care Department admission. Drug overdose produces 4.6 million emergency unit visits and $>80,000$ deaths each year in the USA. ${ }^{1}$ The death rate caused by drug overdose has increased by $137 \%$ since $2000 .{ }^{2}$ Antidepressant self-poisoning is involved in about $20 \%$ of all drug suicides, with the older tricyclic antidepressants (TCAs) showing more severe toxicity than the newer selective serotonin reuptake inhibitors. ${ }^{3}$ One of the most used TCAs in the drug-related deaths is amitriptyline, which exhibits a greater toxicity compared to the other TCAs. Overdose of amitriptyline results in toxic effects in the neurogenic and cardiovascular systems, such as coma, seizure, hypotension, and arrhythmia. ${ }^{4}$ There has been no specific detoxifier for this drug until now. In the last 10 years, intravenous lipid emulsions (ILEs) have been used to treat severe intoxication induced by lipophilic drugs, including TCAs. The intoxications of other drugs such as anesthetics, $\beta$-blockers, parasiticides, and antipsychotics can be alleviated with ILEs. ${ }^{5,6}$ The most likely mechanism of ILE detoxification is the lipid sink effect, the lipophilic drug entrapment into the lipid phase in plasma, which prevents the toxic molecules from 
acting on target organs. The other mechanisms include enhanced mitochondrial fatty acid uptake and elevation of myocardial calcium concentration. ${ }^{7}$

ILEs are composed of triglycerides from soybean oil, egg phospholipids, and glycerol. ${ }^{8}$ The complications associated with the components of ILEs are relatively common. These include fat overload syndrome, fat embolism, hypertriglyceridemia, and egg allergy. ${ }^{9}$ Another concern is that, in some cases, ILEs show no improved resuscitation or even worse survival in experimental and clinical intoxication. ${ }^{10}$ The particle size of ILEs is within the submicron range of $350-450 \mathrm{~nm}$. The formulation optimization and size reduction to nanosized level would be a promising approach to rapidly and extensively entrap the overdosed drugs for detoxification. The large surface area to volume ratio, specific loading capacity, prolonged circulation time, and biosafety of nanomaterials contribute to their possible beneficial effect as antidotes. ${ }^{11}$ We had previously developed the nanoemulsions, which are termed squarticles, that contain squalene as the lipid cores and Pluronic F68/phosphatidylcholines as the emulsifiers for enhancing anti-alopecia drug uptake in hair follicles. ${ }^{12}$ Squalene is a polyunsaturated hydrocarbon with a lipophilic character and high biocompatibility. This nonpolar compound is reported to increase the elimination of xenobiotics due to its affinity to lipophilic molecules. ${ }^{13}$ Squalene exhibits an antidotal effect on theophylline, phenobarbital, cyclophosphamide, arsenic, and lead. ${ }^{14}$ Lack of triglycerides in squarticles reduces the adverse effects elicited by ILEs. Squarticles with a diameter of $<200 \mathrm{~nm}$ may be used as agents for nanodetoxification.

The primary aim of this study was to evaluate if squarticles modified from our previous work showed beneficial potential for scavenging antidepressants. Amitriptyline was used as the model drug because it is a prototypical TCA with a large distribution volume. The impact of nanodetoxification on drug pharmacokinetics and biodistribution is not well understood. The secondary aim of this study was to elucidate this influence by assessing bioimaging and amitriptyline distribution in rats. The possible interaction between squarticles and amitriptyline was examined by drug entrapment, drug release, and isothermal titration calorimetry (ITC) to explore the detoxification mechanisms. We had prepared anionic and cationic nanoemulsions as the variables to investigate their effect on biodistribution and detoxification.

\section{Materials and methods}

\section{Preparation of squarticles}

The lipid phase of anionic squarticles contained squalene $(8 \% \mathrm{w} / \mathrm{v}$ of the final product) and phosphatidylcholines
(Phospholipon ${ }^{\circledR} 80 \mathrm{H}, 2 \%$ ), while the aqueous phase used Pluronic F68 (4.5\%) and water. Octadecylamine (2\%) was added in the lipid phase to produce cationic squarticles. Both phases were separately heated to $85^{\circ} \mathrm{C}$ for $15 \mathrm{~min}$. The two phases were mixed under high-shear homogenization (Pro 250; Pro Scientific, Oxford, CT, USA) at 12,000 rpm for $30 \mathrm{~min}$. Subsequently the mixture was sonicated by VCX 600 (Sonics and Materials) at $25 \mathrm{~W}$ for $25 \mathrm{~min}$. The total volume of the final product was $10 \mathrm{~mL}$. Solid lipid nanoparticles (SLNs) were prepared by the same procedures, but with different lipid phases. The lipid phase of SLNs was a blend of hexadecyl palmitate ( $8 \%$ ) and Phospholipon $80 \mathrm{H}(2 \%)$.

\section{Preparation of liposomes and niosomes}

The amitriptyline entrapment ability of squarticles was compared with that of liposomes and niosomes in this study. Phospholipon $80 \mathrm{H}(2.2 \%)$ and cholesterol $(0.8 \%)$ were dissolved in a mixture of chloroform and ethanol (2:1). The solvent was evaporated in a rotary evaporator at $50^{\circ} \mathrm{C}$, and the traces were removed under a vacuum overnight. The phospholipid thin film was hydrated with water using VCX 600 at $35 \mathrm{~W}$ for $20 \mathrm{~min}$ to produce the liposomes $(10 \mathrm{~mL})$. To prepare the niosomes, Span 60 (0.35\%), Phospholipon $80 \mathrm{H}(0.25 \%)$, cholesterol $(0.35 \%)$, and distearoylphosphatidylethanolamine polyethylene glycol $(0.2 \%)$ were dissolved in the mixture of chloroform and ethanol. The other procedures were the same as the preparation method of liposomes.

\section{Size and zeta potential}

The mean diameter and the zeta potential of the prepared nanosystems were measured by a laser-scattering technique (Nano ZS90; Malvern). The measurement was carried out in the nanosystem concentration after a 100 -fold dilution with water.

\section{Transmission electron microscopy (TEM)}

The morphology of the nanoparticles was monitored using H7500 electron microscopy (Hitachi). One drop (10 $\mu \mathrm{L})$ of the nanosystems was pipetted onto a carbon film-coated copper grid to form a thin-film specimen and then stained with phosphotungstic acid (1\%). The prepared samples were photographed by TEM.

\section{Polarity}

The polarity of the nanosystems was assessed with a fluorescence spectrophotometer (F2500; Hitachi) based on the solvatochromism of Nile red. The nanoparticles were included with Nile red (1 ppm). The emission spectra of 
dye-loaded nanosystems were scanned from 550 to $700 \mathrm{~nm}$. The excitation wavelength for determining the polarity was $546 \mathrm{~nm}$.

\section{Amitriptyline encapsulation by the nanosystems}

For the determination of drug entrapment by the nanosystems, a $3 \mathrm{~mL}$ amitriptyline solution (5 $\mathrm{mM}$ in $20 \%$ propylene glycol/pH 7.4 buffer) was mixed with the nanosystems $(1 \mathrm{~mL})$ in a vial at $37^{\circ} \mathrm{C}$. The addition of propylene glycol to the aqueous solution aimed to assure complete solvation of amitriptyline in the vehicle. The mixture was agitated by a stir bar at $600 \mathrm{rpm}$. At determined intervals of 1, 2, 4, 6, and $8 \mathrm{~h}$, the mixture $(0.3 \mathrm{~mL})$ was withdrawn and centrifuged at $48,000 \times g$ and $4^{\circ} \mathrm{C}$ for $40 \mathrm{~min}$. The precipitate was dissolved by Triton X-100 to determine the drug entrapment ratio in the nanoparticles. Amitriptyline concentration was detected by high-performance liquid chromatography (HPLC) method, as described previously. ${ }^{15}$ ILEs (Intralipid ${ }^{\circledR}$ ) were also tested in this experiment.

The maximum loading of amitriptyline in the nanoparticles was evaluated by an ultracentrifugation technique. Amitriptyline $(10 \mathrm{mg}$ ) was added into the lipid phase to produce the nanosystems $(10 \mathrm{~mL})$. The nanoformulations were centrifuged at $48,000 \times g$ and $4^{\circ} \mathrm{C}$ for $40 \mathrm{~min}$. The supernatant and the precipitate were separated and analyzed by HPLC to calculate the encapsulation percentage of the initial amount of amitriptyline added.

\section{Isothermal titration calorimetry}

ITC (MicroCal ${ }^{\circledR}$ iTC200; GE Healthcare) was used to rate the interaction between amitriptyline and the nanoparticulate surface. An initial injection $(0.4 \mu \mathrm{L})$ and subsequent 19 injections $(2 \mu \mathrm{L})$ of $5 \mathrm{mM}$ amitriptyline were infused into the sample cell, which was filled with the nanosystems at $37^{\circ} \mathrm{C}$. The nanosystems were stirred at $1,000 \mathrm{rpm}$. The caloric data were collected at $2.5 \mathrm{~min}$ intervals for $50 \mathrm{~min}$. The titration data and the binding plot were computed with a one-site binding model.

\section{Amitriptyline release from the nanosystems}

The nanosystems were centrifuged at $48,000 \times g$ and $4{ }^{\circ} \mathrm{C}$ for $40 \mathrm{~min}$ to withdraw the unencapsulated amitriptyline in the supernatant. The nanoparticles in precipitate were reconstituted by adding water to the pellets to achieve an amitriptyline concentration of $0.5 \mathrm{mg} / \mathrm{mL}$. Amitriptyline release from the nanosystems was conducted using Franz diffusion cells, as in a previous study. ${ }^{16}$ HPLC was used to quantify amitriptyline release into the receptor compartments of the Franz cells.

\section{Animals}

Male Sprague Dawley rats weighing 350-400 g were obtained from BioLasco (Taipei, Taiwan). The experiments were approved and performed in strict accordance with the recommendations set forth in the Guidelines for the Institutional Animal Care and Use Committee of Chang Gung University.

\section{In vivo and ex vivo bioimaging}

The rats were randomly divided into three groups with six animals in each. Group 1 received intravenous 1, $1^{\prime}$-dioctadecyl3,3,3', $3^{\prime}$-tetramethyl-indotricarbocyanine iodide (DiR; $50 \mu \mathrm{g} / \mathrm{mL}$ in dimethyl sulfoxide:Tween 80 : saline $=2: 1: 2$ ) as the near-infrared (NIR) dye to monitor the real-time and organ distribution. Group 2 and Group 3 received intravenous anionic and cationic squarticles containing DiR, respectively. The nanoformulations were diluted by water to $20 \%$. The rats were anesthetized using Zoletil ${ }^{\circledR} 50(60 \mathrm{mg} / \mathrm{kg})$. A bolus of $1.5 \mathrm{~mL} / \mathrm{kg}$ free $\mathrm{DiR}$ or nanosystems was administered into the femoral vein for $1 \mathrm{~min}$, followed by a constant rate infusion of $6 \mathrm{~mL} / \mathrm{kg} / \mathrm{h}$ until the end of experiment $(90 \mathrm{~min})$. The real-time imaging of the rats was visualized at the determined intervals using Pearl ${ }^{\circledR}$ Impulse (Li-Cor). To monitor the ex vivo bioimaging, DiR was replaced by the NIR dye of iFluor $^{\circledR} 790$ to be doped into the nanoparticles for obtaining better resolution of the peripheral organ imaging. The rats were sacrificed at $90 \mathrm{~min}$ after iFluor 790 administration. The organs were excised for monitoring free iFluor 790 or nanoparticle distribution.

\section{Pharmacokinetics and biodistribution of amitriptyline}

Ten anesthetized rats received intravenous amitriptyline, followed by a continuous infusion of saline, squarticles, or ILEs for examining amitriptyline pharmacokinetics and organ distribution. A $5 \mathrm{mg} / \mathrm{kg}$ dose of amitriptyline was injected as an infusion over 15 min into the right femoral vein. The amitriptyline was then allowed to distribute into the organs for $15 \mathrm{~min}$ after the end of infusion. The rats received a $1.5 \mathrm{~mL} / \mathrm{kg}$ bolus of saline or antidotes ( $20 \%$ dilution before injection) for $1 \mathrm{~min}$ and then a $6 \mathrm{~mL} / \mathrm{kg} / \mathrm{h}$ infusion for $89 \mathrm{~min}$. Immediately after beginning the antidote administration, blood samples were collected from the left femoral vein at 5, 15, 30, 60, and $90 \mathrm{~min}$. At $90 \mathrm{~min}$ after the antidote injection, we sacrificed the rats to remove the organs. The blood samples were centrifuged at $2,000 \times g$ at $4{ }^{\circ} \mathrm{C}$ for 
10 min to obtain plasma. The plasma $(100 \mu \mathrm{L})$ was mixed with acetonitrile $(300 \mu \mathrm{L})$ for $10 \mathrm{~min}$ and then centrifuged at $8,000 \times g$ and $4{ }^{\circ} \mathrm{C}$. The supernatant was filtered across the membrane with a pore size of $0.45 \mu \mathrm{m}$. The amount of amitriptyline in the plasma was detected by HPLC. The harvested organs were weighed, cut, and suspended in methanol (1 mL). MagNA Lyser (Roche, East Lyme, CT, USA) was utilized to homogenize the organs. After centrifugation, the supernatant $(300 \mu \mathrm{L})$ was evaporated by a vacuum pump. Nine hundred microliters of acetonitrile was used to dissolve the samples. The amitriptyline concentration in the samples was then quantified by HPLC.

\section{Pharmacodynamic assay}

In this study, the protocol of the amitriptyline and antidote administration was the same as in the pharmacokinetic study. Mean arterial pressure (MAP) was detected by PowerLab/8SP cardiac monitor (AD Instruments, Colorado Springs, CO, USA). The probe was inserted into the left femoral vein for monitoring. The sample number of each group was six.

\section{Survival rate}

A high dose of amitriptyline $(11.5 \mathrm{mg} / \mathrm{kg})$ was intravenously infused into the femoral vein over $10 \mathrm{~min}$. Then, we injected the antidotes at a bolus of $1.5 \mathrm{~mL} / \mathrm{kg}$ for $1 \mathrm{~min}$, followed by a constant infusion rate of $6 \mathrm{~mL} / \mathrm{kg} / \mathrm{h}$. The rat survival was monitored for $120 \mathrm{~min}$ after amitriptyline treatment $(n=12)$.

\section{Statistical analysis}

The data were presented as mean and SD. The difference in the data related to the different treatment groups was analyzed using the Kruskal-Wallis test. Dunn's test was used as the post hoc test for checking individual differences. Significance was indicated as $p<0.05$.

\section{Results}

\section{Size and zeta potential}

The particulate size, surface charge, and other physicochemical properties are essential parameters for correlating nanoparticle affinity and capacity to the toxicants. The hydrodynamic diameter, polydispersity index, and zeta potential of the tested nanoparticles are summarized in Table 1. The nanoformulations varied in terms of the size and surface charge. The diameters of the anionic and cationic squarticles were found to be 97 and $122 \mathrm{~nm}$, respectively. The polydispersity index was detected to be below 0.3 , indicating the narrow distribution of the particle population. The zeta potential of anionic squarticles was highly
Table I The characterization of nanosystems by particulate size, PDI, and zeta potential

\begin{tabular}{llll}
\hline Formulation & Size $(\mathbf{n m})$ & PDI & Zeta potential $(\mathbf{m V})$ \\
\hline Anionic squarticles & $97.19 \pm 0.19$ & $0.30 \pm 0.02$ & $-35.55 \pm 1.65$ \\
Cationic squarticles & $122.23 \pm 0.55$ & $0.21 \pm 0.01$ & $-27.60 \pm 0.44$ \\
SLNs & $190.63 \pm 0.19$ & $0.20 \pm 0.01$ & $-32.33 \pm 0.12$ \\
Liposomes & $139.07 \pm 2.60$ & $0.14 \pm 0.02$ & $-42.17 \pm 0.15$ \\
Niosomes & $92.59 \pm 0.41$ & $0.24 \pm 0.01$ & $-5.32 \pm 0.91$ \\
\hline
\end{tabular}

Note: Each value represents the mean $\pm S D(n=3)$

Abbreviations: PDI, polydispersity index; SLNs, solid lipid nanoparticles.

negative. Cationic octadecylamine could be intercalated in the oil-water interface to produce positive zeta potential. SLNs are the nanoparticles with solid lipid as the core. SLNs showed larger size $(191 \mathrm{~nm})$ than the squarticles. The zeta potential of SLNs was comparable to that of anionic squarticles. Both liposomes and niosomes are nanovesicles with a bilayer structure. Liposomes and niosomes were found to have sizes of 139 and $93 \mathrm{~nm}$, respectively. The presence of phosphatidylcholines in the lipid bilayers contributed to the negative surface charge of liposomes $(-42 \mathrm{mV})$, whereas the surface charge of niosomes was near neutral due to the main bilayer material of nonionic surfactant. The size and zeta potential of ILEs were $430 \mathrm{~nm}$ and $-17.3 \mathrm{mV}$, respectively, according to a previous investigation and application note from Beckman Coulter. ${ }^{17}$ ILEs can be categorized as submicron emulsions.

\section{Amitriptyline entrapment, morphology, and polarity of the nanosystems}

To study the ability of the nanosystems to scavenge amitriptyline, the drug solution was incubated with the nanoformulations for examining the entrapment percentage, as shown in Figure 1A. Squarticles and ILEs displayed a fast and comparable entrapment. A saturation level was achieved as the percentage of entrapped amitriptyline reached $40 \%-50 \%$. The drug-entrapped effect of SLNs was 2-fold lower than that of squarticles. The least entrapment was found to be of liposomes and niosomes, showing only $16 \%$ and $12 \%$ amitriptyline load after $8 \mathrm{~h}$ of incubation.

Figure 1B shows the morphology of anionic and cationic squarticles observed under TEM. The nanoparticles were spherical or nearly spherical with a smooth surface. The nanoparticles were well distributed as individual particles. The sizes shown in the images were 100-200 nm, which were slightly larger than those measured in the hydrodynamic state. Figure $1 \mathrm{C}$ depicts the emission spectra of Nile red in the squarticles. The fluorescence of Nile red is quenched in the environment with higher polarity. The cationic squarticles 

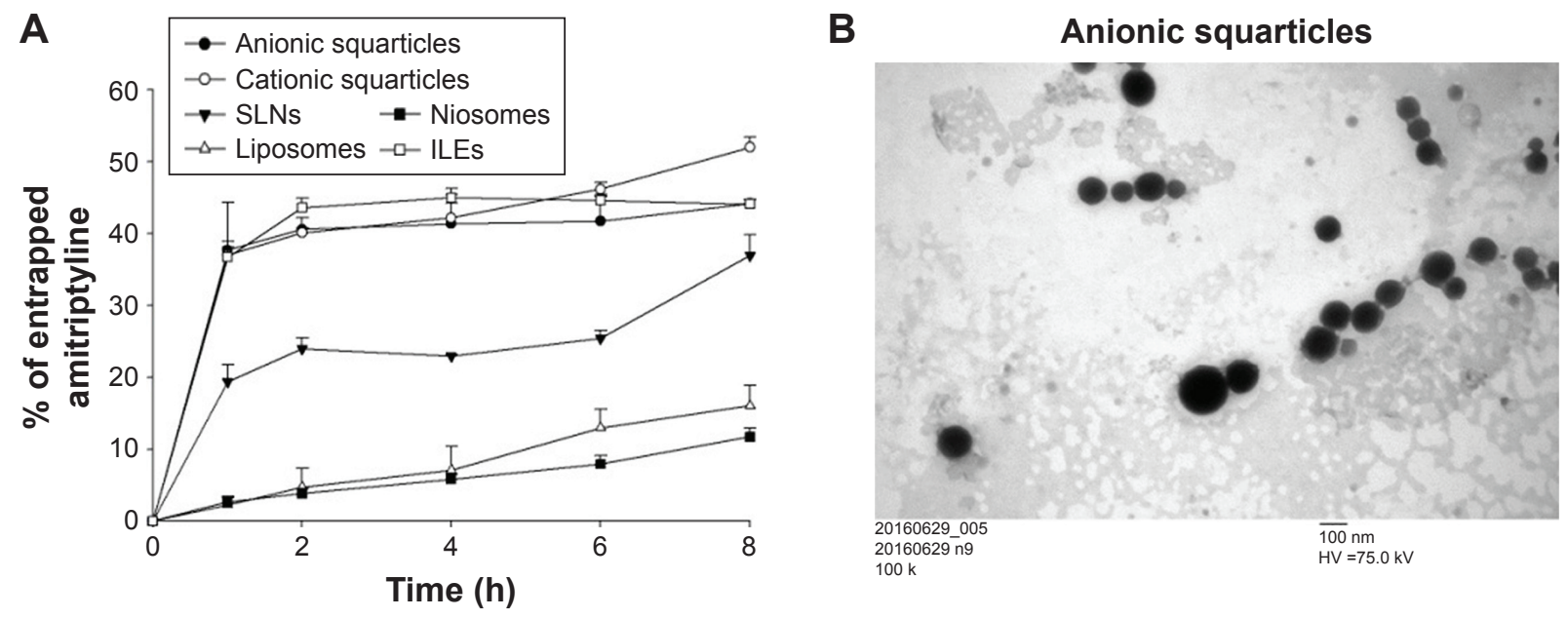

\section{Cationic squarticles}
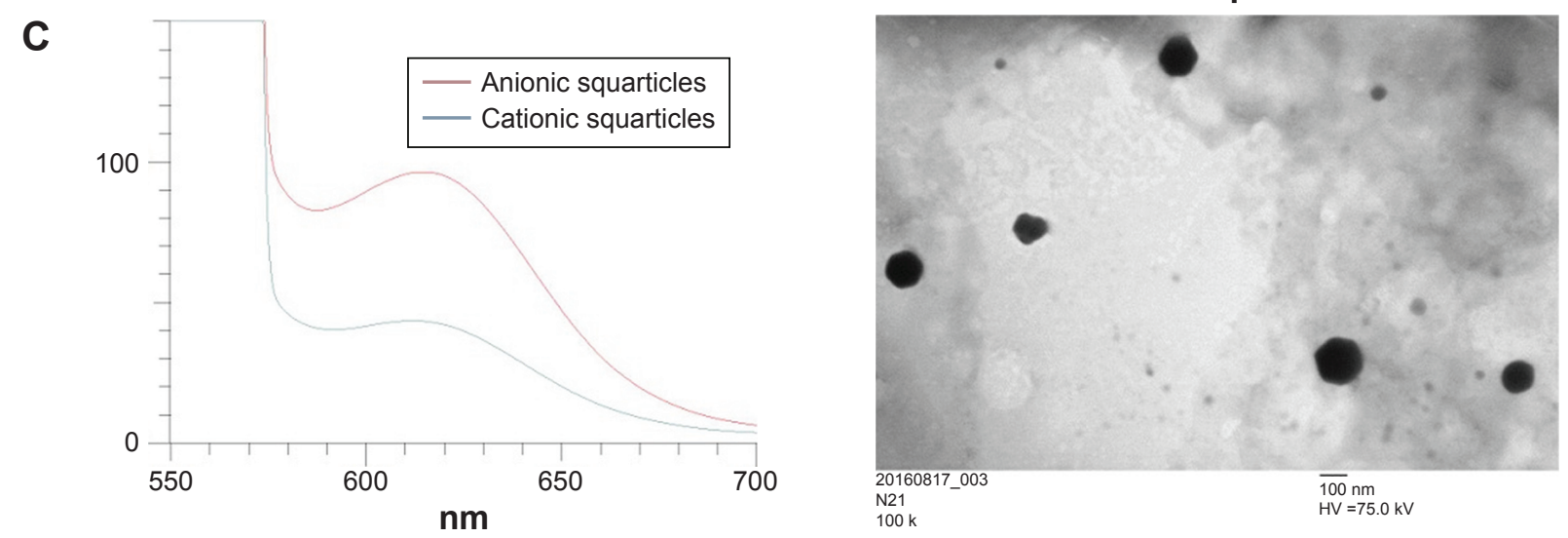

Figure I Amitriptyline entrapment, morphology, and polarity of the nanosystems.

Notes: (A) Amitriptyline entrapment percentage into the nanosystems as a function of time; (B) the morphology of anionic and cationic squarticles viewed by TEM; and (C) the polarity of anionic and cationic squarticles determined by the solvatochromism of Nile red. Each value represents the mean and SD ( $\mathrm{n}=4$ ).

Abbreviations: ILEs, intravenous lipid emulsions; SLNs, solid lipid nanoparticles; TEM, transmission electron microscopy.

revealed a weaker emission compared with the anionic squarticles. This suggests a more hydrophilic nature of squarticles after the incorporation of cationic amphiphile.

\section{Amitriptyline's interaction with nanoparticulate surface and release from the nanosystems}

We next evaluated the possible interaction between amitriptyline and squarticles. ILEs were also tested for comparison. ITC characterizes the underlying thermodynamics of the physicochemical affinity. As Figure 2A shows, the positive injection signal in the case of the anionic squarticles demonstrates an endothermic interaction. A contrary injection signal was observed for cationic squarticles, suggesting an exothermic process. The association constant $\left(K_{\mathrm{a}}\right)$ was found to be 28,700 and 5,010 for anionic and cationic squarticles, respectively, based on the titration curve. The increased $K_{\text {a }}$ suggests an enhanced specific binding between the drug and nanoparticles. The heat profile could not be sufficiently fitted by the interaction between amitriptyline and ILEs, indicating a negligible affinity of amitriptyline to the surface of ILEs.

To check the maximal loading capability of amitriptyline into squarticles, the amitriptyline encapsulation efficiency was examined, as shown in Figure 2B. Amitriptyline was incorporated in the lipid phase of the squarticles in the nanosystem preparation procedure for the following determination. Since commercially available ILEs were purchased as a final product, it was impossible for us to maximally load amitriptyline into ILEs during the product preparation procedure. Thus, we did not test the loading capacity of amitriptyline to ILEs in this experiment. The drug encapsulation of cationic squarticles (36\%) was slightly, but significantly greater than that of anionic squarticles (32\%). ILEs were not tested in this experiment, since the maximal drug loading could not be obtained as the antidote formulation had already been prepared. The entrapment of toxicants by 
A

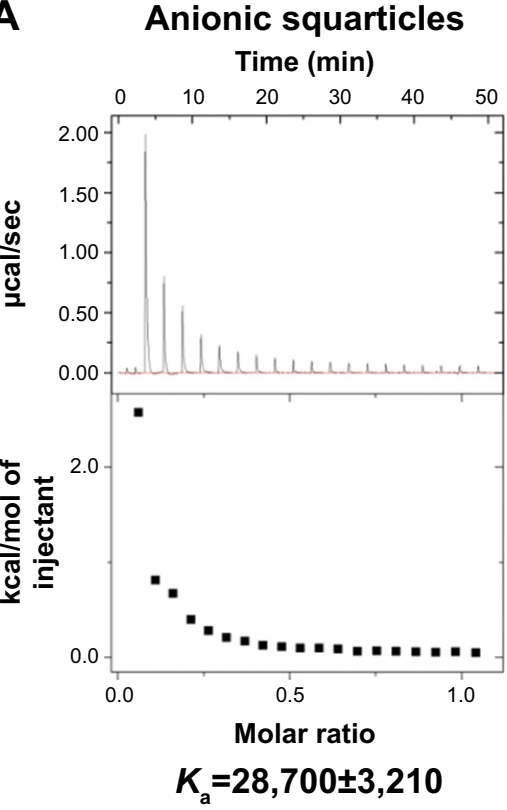

B

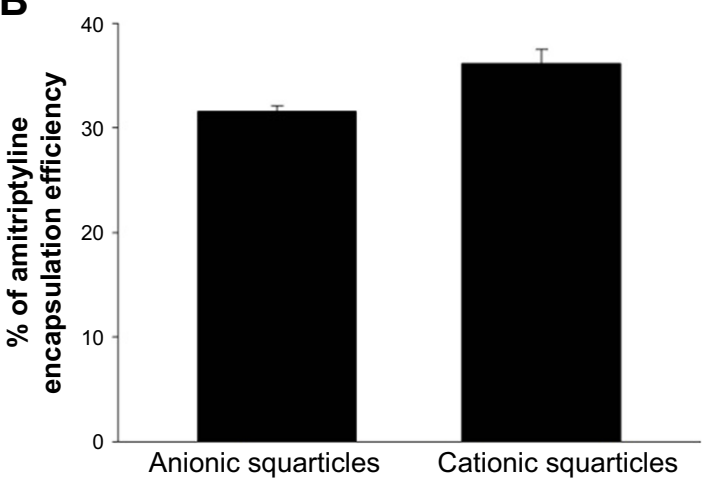

Cationic squarticles

Time ( $\mathrm{min})$

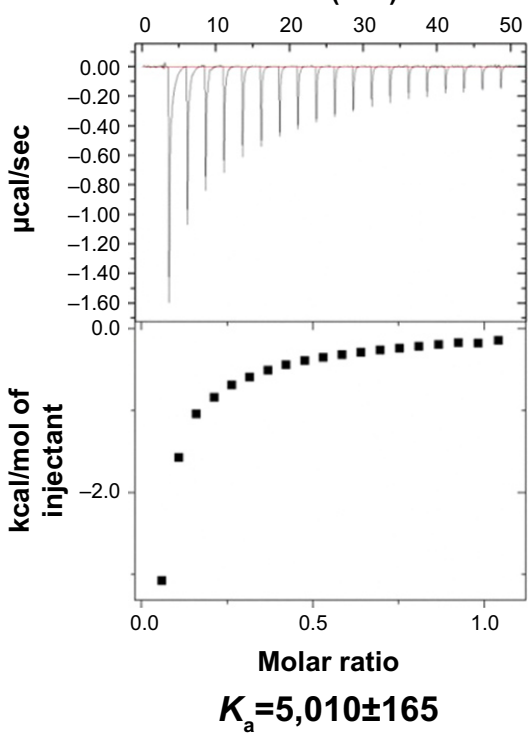

ILEs

Time ( $\mathrm{min})$

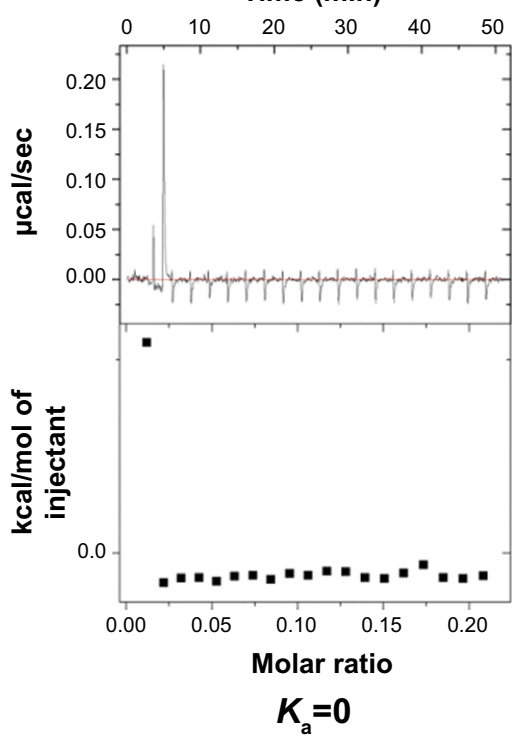

C

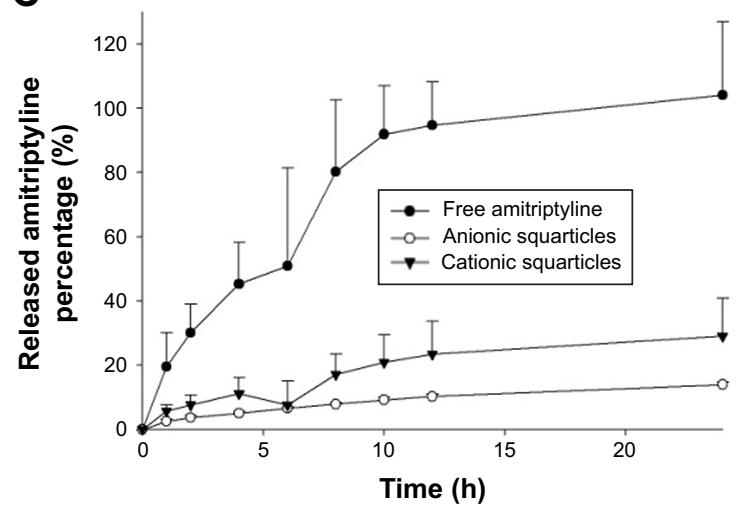

Figure 2 Interaction of amitriptyline with nanoparticulate surface and release from the nanosystems.

Notes: (A) Isothermal titration calorimetry data from the titration of amitriptyline into squarticles and ILEs; (B) the encapsulation efficiency of amitriptyline in squarticles; and $(\mathbf{C})$ the release of amitriptyline from squarticles. Each value represents the mean and SD $(n=4)$.

Abbreviations: ILEs, intravenous lipid emulsions; $K_{\mathrm{a}}$, association constant.

the antidotes can be reversible. We analyzed the possible release of amitriptyline from the squarticles. In this assay, amitriptyline was completely encapsulated in the nanoparticles by reconstituting the ultracentrifuged nanoparticle pellet after free drug removal. Figure $2 \mathrm{C}$ depicts a slower amitriptyline release from the squarticles than that from the control solution. Amitriptyline exhibited an initial burst from the control vehicle and then gradually leveled off after $10 \mathrm{~h}$. Squarticles showed a sustained amitriptyline release. The anionic squarticles offered 2-fold less drug release than the cationic ones.

\section{In vivo and ex vivo bioimaging}

To check the biofate of squarticles in the rats, the biodistribution of the nanoparticles was assayed by real-time and ex vivo bioimaging. The residence of squarticles in circulation was observed using DiR as the probe. Figure $3 \mathrm{~A}$ illustrates the DiR intensity as a function of time after the intravenous infusion of free DiR and squarticles. The probe intensity of all images was calibrated by the DiR-loaded formulation intensity for an impartial comparison. There was no NIR signal in the vessels before administration. A continuous infusion was performed throughout $90 \mathrm{~min}$. The DiR intensity was increased following the increase in time for the control vehicle. The morphology of the vessels was clearly visualized after $30 \mathrm{~min}$. A similar trend was found in the group receiving anionic squarticles, suggesting a prolonged circulation residence. In contrast, infusion of cationic squarticles led to an insignificant accommodation in the vessels. The optical signal was mainly observed in the region of the liver. 


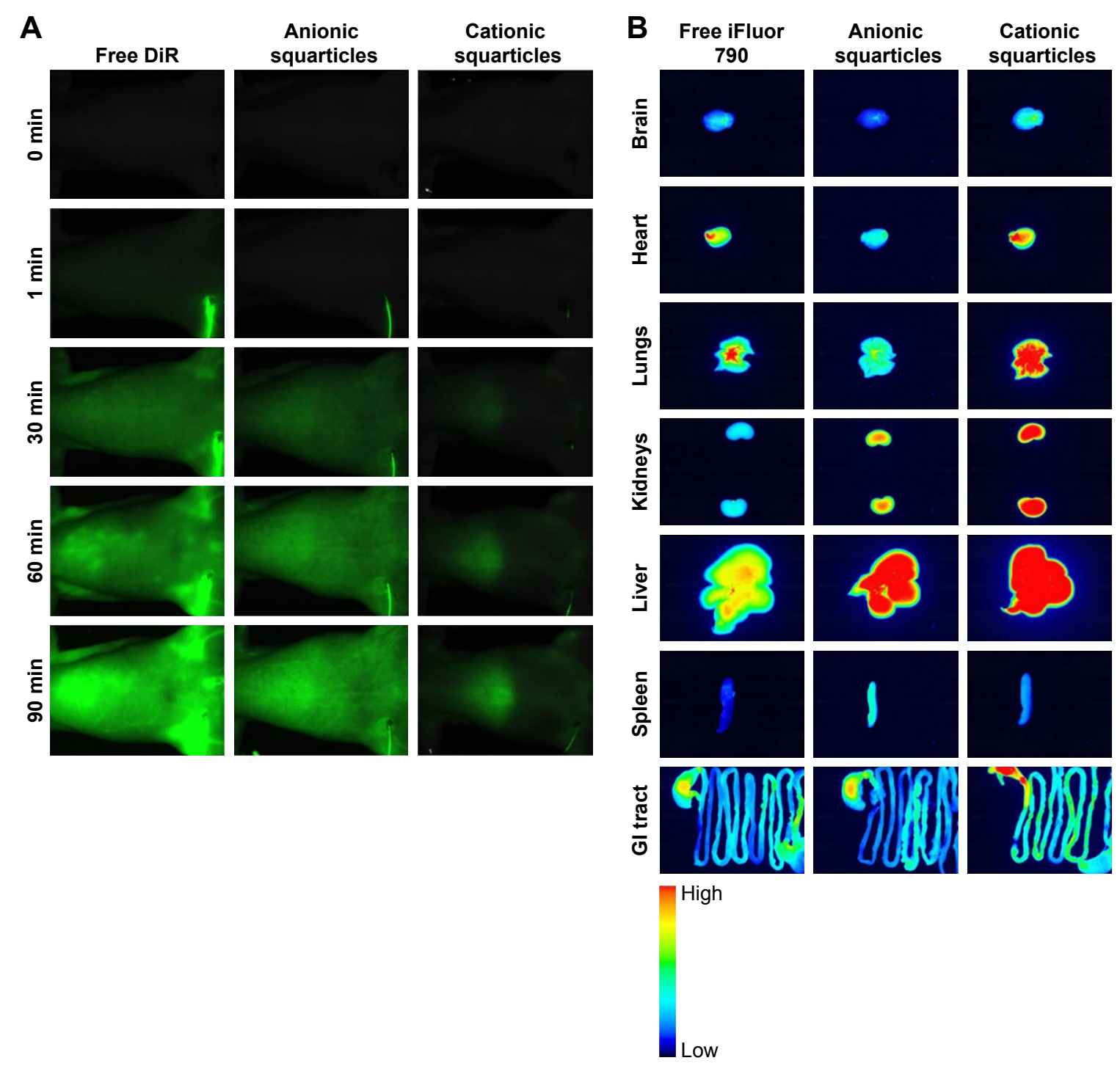

Figure 3 In vivo and ex vivo bioimaging of rats receiving free dye and dye-loaded squarticles.

Note: (A) Real-time imaging for viewing the residence in circulation and (B) ex vivo bioimaging of the organs of the representative animals at the end of the experiment. Abbreviations: DiR, I, I'-dioctadecyl-3,3,3',3'-tetramethyl-indotricarbocyanine iodide; GI tract, gastrointestinal tract.

The intensity in the liver progressively increased until the end of the experiment.

The iFluor 790 probe was selected as the dye for ex vivo bioimaging due to the high sensitivity of this probe for peripheral organ imaging. Free iFluor 790 principally accumulated in the heart, lungs, and liver, as presented in Figure 3B. The uptake of the free dye by spleen could be neglected. Strong intensity for anionic squarticles was seen mainly in the liver, followed by the kidneys and lungs. The brain showed minor accumulation of anionic squarticles. Compared with anionic squarticles, the cationic nanoformulations resulted in an extensive residence in most of the organs. This can explain the shorter circulation period of the cationic squarticles compared with the anionic ones. The percentage of iFluor 790 signal in different organs detected by intravenous infusion of free dye and squarticles is summarized in Table 2. In the rats receiving anionic squarticles, the accumulation in the liver (33\%) was much greater than in the other organs. Although the real-time imaging demonstrated a significant uptake of cationic squarticles in the liver, the highest portion of nanoparticle accumulation was found in the lungs (27\%). The NIR percentage of the liver and kidneys was comparable $(21 \%)$.

\section{Pharmacokinetics and biodistribution of amitriptyline}

We assessed the effect of squarticles on amitriptyline intoxication by intravenous injection. Most of the TCA intoxication occurs from oral ingestion, followed by the absorption into circulation, tissue biodistribution, and metabolism. Our goal 
Table 2 The percentage of near-infrared signal in different organs of rats by intravenous injection of free iFluor 790 and the squarticles containing iFluor 790

\begin{tabular}{llll}
\hline Organ & $\begin{array}{l}\text { Free iFluor } \\
790\end{array}$ & $\begin{array}{l}\text { Anionic } \\
\text { squarticles }\end{array}$ & $\begin{array}{l}\text { Cationic } \\
\text { squarticles }\end{array}$ \\
\hline Brain & $6.71 \pm 1.50$ & $3.76 \pm 0.41$ & $4.11 \pm 0.36$ \\
Heart & $22.89 \pm 1.82$ & $11.06 \pm 2.69$ & $9.79 \pm 1.26$ \\
Lungs & $22.27 \pm 5.04$ & $15.25 \pm 2.47$ & $27.35 \pm 3.26$ \\
Kidneys & $10.61 \pm 0.95$ & $21.03 \pm 3.99$ & $21.13 \pm 1.70$ \\
Liver & $23.31 \pm 0.89$ & $33.18 \pm 3.63$ & $21.82 \pm 2.68$ \\
Spleen & $3.54 \pm 0.58$ & $9.97 \pm 2.17$ & $9.17 \pm 0.24$ \\
Gl tract & $7.66 \pm 0.72$ & $5.74 \pm 0.51$ & $6.64 \pm 0.10$ \\
\hline
\end{tabular}

Note: Each value represents the mean $\pm S D(n=6)$.

Abbreviation: Gl tract, gastrointestinal tract.

was not to simulate oral absorption. We aimed to mimic a condition wherein amitriptyline was already absorbed from the gastrointestinal tract and distributed into the organs. This would mimic the post-absorption stage of acute oral intoxication. The protocol of the pharmacokinetic and pharmacodynamic studies is depicted in Figure 4A. Amitriptyline was infused continuously for $15 \mathrm{~min}$ and then allowed to distribute into the organs for the next $15 \mathrm{~min}$. After biodistribution, squarticles or ILEs were infused with both bolus and continuous doses. The total duration of the experiment was $120 \mathrm{~min}$. Figure 4B shows the concentration of amitriptyline in the plasma after antidote administration. The plasma drug concentration at the end of infusion was comparable for all tested groups $(\sim 6 \mu \mathrm{g} / \mathrm{mL})$. The drug concentration declined rapidly in the saline control, implying fast distribution. The plasma in the groups of anionic squarticles and ILEs contained more amitriptyline, compared to the control. In contrast, the cationic squarticles did not increase the drug concentration at 15 min post-infusion. The area under the curve for saline, anionic squarticles, cationic squarticles, and ILEs was 19.82, 48.73, 13.91, and $34.48 \mu \mathrm{g} \cdot \mathrm{min} / \mathrm{mL}$, respectively.

Figure 4C shows amitriptyline distribution in different organs at the end of the experiment (120 min). Higher fractions of free amitriptyline (saline control) were found in the brain, lungs, and spleen. Antidote treatment resulted in less drug retention in these three organs. Anionic squarticles and ILEs revealed a greater decrease of amitriptyline in the brain than the cationic squarticles. The drug concentration in the lungs and spleen was comparable for the three antidote formulations. Anionic squarticles and ILEs, but not cationic squarticles, could reduce amitriptyline accumulation in the heart and kidneys by 2-fold. There was no amitriptyline in the liver which received saline. The antidote application increased the amount of liver amitriptyline, indicating a possible metabolism for detoxification. We further analyzed the accumulation ratio of amitriptyline between rapidly perfused organs and plasma area under the curve (Figure 4D). The organ-to-plasma ratio was significantly lower in the anionic squarticles than in the other groups, suggesting efficient removal of amitriptyline from the organs and return to circulation. ILEs demonstrated comparable brain-to-plasma and lungs-to-plasma ratios to anionic squarticles.

\section{Pharmacodynamic assay}

The rats were instrumental in determining the MAP. The measurement was recorded for $120 \mathrm{~min}$, as illustrated in Figure 4E. The MAP was similar among the tested groups before the antidote application. The MAP decreased from $\sim 120$ to $\sim 90 \mathrm{mmHg}$ after amitriptyline injection at $30 \mathrm{~min}$. The blood pressure returned to the baseline within $10 \mathrm{~min}$ in the group receiving anionic squarticles, suggesting an efficient improvement of drug-induced cardiotoxicity. This recovery to baseline was also obtained in the group receiving ILEs after a $90 \mathrm{~min}$ infusion. Saline could ameliorate the hypotensive effect with a slower and lesser recovery, compared to anionic squarticles and ILEs. The MAP in the rats that received cationic squarticles was not restored during the continuous infusion.

\section{Survival rate}

A lethal dose of amitriptyline $(11.5 \mathrm{mg} / \mathrm{kg})$ was administered to rats in order to detect the survival rate. The protocol for administering the drug and antidotes is given in Figure 5A. Figure 5B shows the Kaplan-Meier curve comparing the survival duration and mortality. The intoxicated rats with saline treatment had a $25 \%$ survival percentage within 8 min after saline infusion. The antidotes could enhance their survival, with the anionic squarticles displaying the highest rate $(75 \%)$. The survival rates for the cationic squarticles and ILEs were $33 \%$ and $58 \%$, respectively. There was no further mortality in all groups at $30 \mathrm{~min}$ after infusion of the antidotes. These surviving rats lived to the end time point (120 min).

\section{Discussion}

The investigation herein focused on the development of detoxifying agents to capture the toxicant drugs. Nanodetoxification can be an efficient strategy for intoxication treatment. Lipid nanoparticles that are mainly composed of an amphiphilic shell and a lipophilic core are of interest in seeking to sequester the toxicants because the characteristics 
A

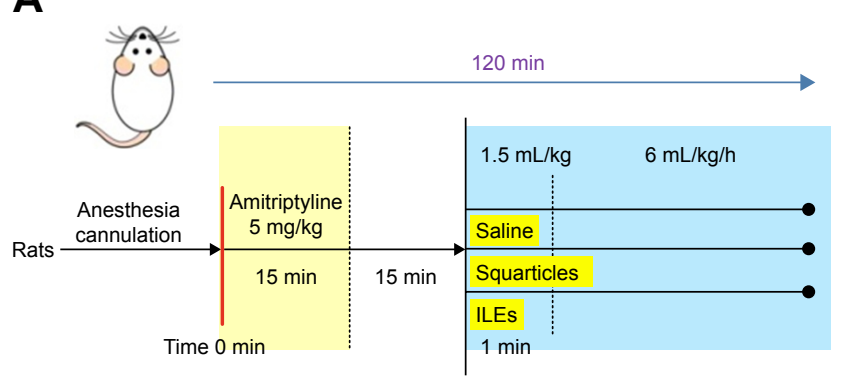

B

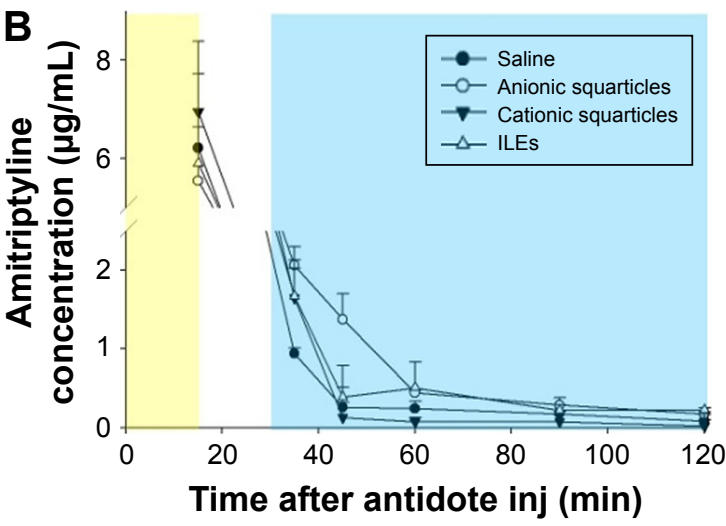

C

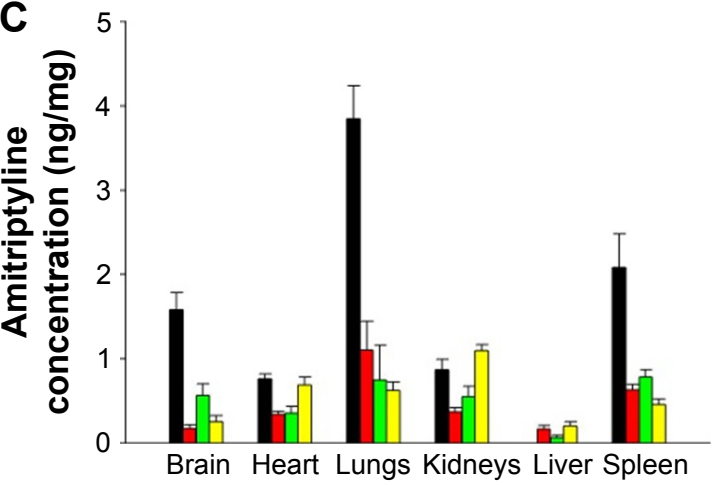

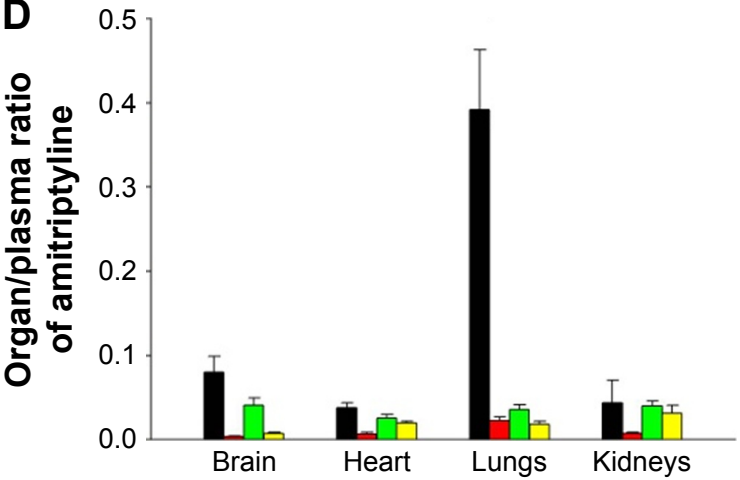

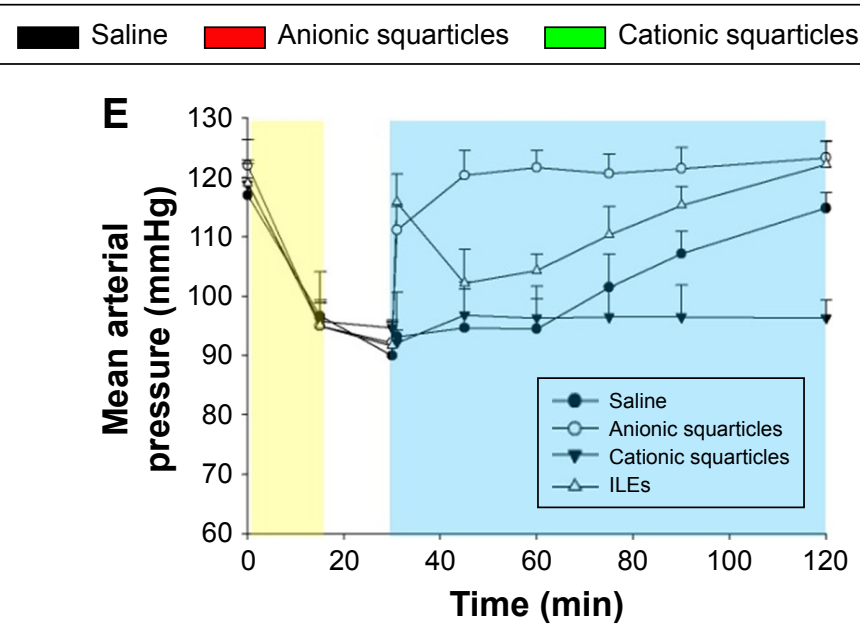

Figure 4 Pharmacokinetic and pharmacodynamic evaluations of the effect of squarticles and ILEs on amitriptyline intoxication.

Notes: (A) The experimental protocol; (B) concentration of amitriptyline in the plasma after intravenous administration of squarticles and ILEs; (C) biodistribution of amitriptyline in the peripheral organs after intravenous administration of squarticles and ILEs; (D) the organ/plasma ratio of amitriptyline concentration after intravenous administration of squarticles and ILEs; and (E) the mean arterial pressure of amitriptyline-intoxicated rats after intravenous administration of squarticles and ILEs. Each value represents the mean and SD ( $n=10$ for pharmacokinetic study and $n=6$ for pharmacodynamic study).

Abbreviations: ILEs, intravenous lipid emulsions; inj, injection.

of this nanoparticle type allow them to be circulated in the hydrophilic environment while entrapping lipophilic molecules. TCAs are heterocyclic molecules with a lipophilic tricyclic scaffold and a hydrophilic chain. These drugs are able to interact with surfactant membranes and lipid bilayers. ${ }^{18}$ An important role of the detoxifying agent is that it can carry the toxicants to be metabolized or excreted after entrapment.
The charged nanoparticles, but not neutral ones, easily interact with serum proteins, promoting clearance in the reticuloendothelial system (RES). ${ }^{19}$ For this reason, we only developed anionic and cationic squarticles in this study. Our results demonstrated that amitriptyline was sequestered to a great amount by the intravenously infused squarticles, especially the anionic nanosystems, which prevented reduction 
A

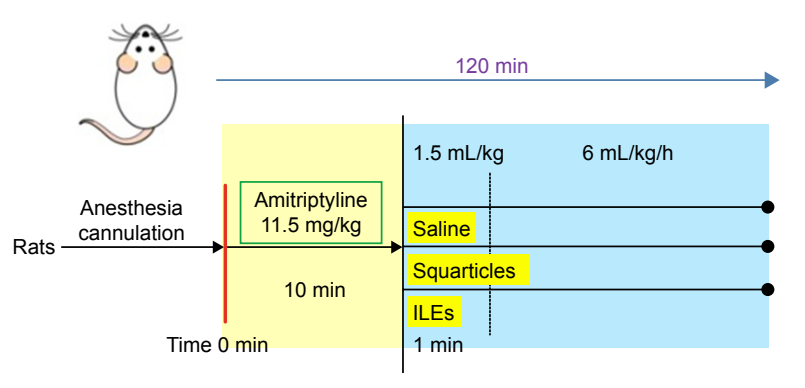

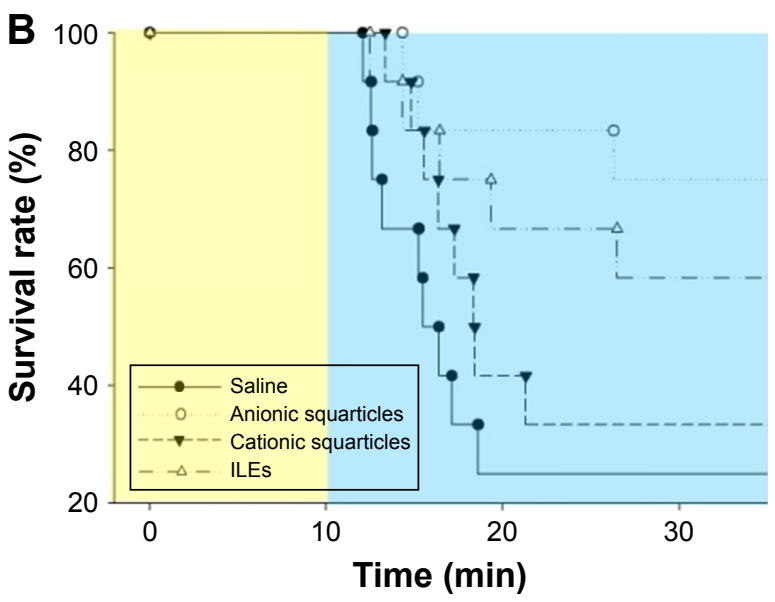

Figure 5 Effect of intravenous administration of squarticles and ILEs on the survival rate of rats receiving overdose of amitriptyline. Notes: (A) The experimental protocol and (B) Kaplan-Meier survival curve of the rats. Each value represents the mean and SD ( $n=12$ ). Abbreviation: ILEs, intravenous lipid emulsions.

of MAP and increased the survival rate in the intoxicated rats. This study certifies the potential of squarticles as a novel nanoformulation for TCA scavenging.

The result of entrapment showed that squarticles and ILEs are principally capable of absorbing amitriptyline, followed by SLNs, liposomes, and niosomes. The maximum amitriptyline entrapment percentage of $\sim 45 \%$ by ILEs approximated the in vitro amitriptyline extraction by ILEs (47\%) reported by French et al. ${ }^{20}$ Fast toxicant transport from the environment to nanoparticles is critical for nanodetoxification. ${ }^{21}$ Squarticles exhibited a quick and effective entrapment to sequester amitriptyline. A previous study ${ }^{1}$ demonstrated comparable drug sequestration by the liposomes and ILEs for drug overdose treatment. This was not the case in our study, since the nanovesicles with a bilayer structure including liposomes and niosomes failed to entrap amitriptyline. Amitriptyline is a weak base with a dissociation constant value of 9.4. In the physiologic condition of $\mathrm{pH} \leq 7.4$, amitriptyline is positively ionized to a high degree. Although the anionic liposomes can capture the cationic drugs within the bilayers, this interaction damages the phospholipid ordering and causes leakage of the entrapped components. ${ }^{22}$ The toxicants are extracted from the aqueous environment through adsorption at the nanoparticulate surface. The extraction can also occur by partitioning the toxicants into the interior core. Lipophilic amitriptyline was difficult to partition into the aqueous core of liposomes and niosomes. SLNs with a crystalline lipid core showed less drug entrapment than the squarticles, even though the emulsifier shell material was the same as that of squarticles. These results suggest that the nanoparticulate core was vital in governing the drug sequestration.

The interaction of amitriptyline with the membrane of squarticles and ILEs was examined by the ITC binding isotherm.
Amitriptyline had a higher affinity to anionic squarticles than to cationic squarticles. This result is explained on the basis of the electrostatic interaction due to the opposite charges between amitriptyline and anionic squarticles. Another observation was that both squarticle types revealed different thermal processes. Anionic and cationic nanoparticles showed endothermic and exothermic events, respectively, indicating the different binding modes for the two nanoformulations. The different modes do not affect the comparison of affinity and $K_{\mathrm{a}}$. The results of entrapment and ITC demonstrate that amitriptyline not only partitioned into the core of squarticles but was also adsorbed onto the nanoparticulate surface. Although the ILEs also displayed a negative charge on the surface, no interaction was found between amitriptyline and ILEs. This suggests that the zeta potential on the particulate surface was not the sole factor predominating amitriptyline extraction. This inference can be confirmed by the low drug entrapment of SLNs and liposomes with negative charge. Since amitriptyline could not interact with the ILE surface, the high drug entrapment of ILEs may be due to the fast and facile entrance of amitriptyline into the lipid core. The submicron particles of ILEs provided a large lipid reservoir in which amitriptyline could be included.

It is possible that the ILEs lose sequestered amitriptyline after entrapment. ${ }^{23}$ The rapid release can result in poor detoxification. This phenomenon is worse in the plasma since the particles suffer from protein and enzymatic erosion in the circulation. The amount of amitriptyline released from the squarticles was limited. The minor release of anionic nanosystems compared to cationic ones indicates a firm emulsifier membrane formed by the anionic squarticles. Nile red emission showed a higher lipophilicity of anionic squarticles compared to cationic squarticles. The nanoparticles with 
higher lipophilicity were capable of holding more lipophilic compounds with less leakage.

Toxicity from TCAs commonly occurs within $2 \mathrm{~h}$ after ingestion. At this time, an abundant amount of TCAs is distributed into the organs. The current recommendation for $20 \%$ ILE therapy is a bolus of $1.5 \mathrm{~mL} / \mathrm{kg}$ within $1 \mathrm{~min}$, followed by a continuous infusion. ${ }^{24}$ Our protocol resembled this recommendation. Amitriptyline mainly causes toxicity in the central nervous system and heart, leading to seizure and hypotension. ${ }^{25}$ Our results demonstrated that anionic squarticles are beneficial to detoxification by the rerouting of amitriptyline from the peripheral organs to the circulation. The hypotension and survival rate could be ameliorated by the nanoantidotes; this is attributed to the electrostatic binding and lipophilic affinity of anionic nanoformulations to the overdosed drug. Amitriptyline is rapidly and facilely distributed from the plasma into the organs. Intravenous application of ILEs results in the extraction of lipophilic drugs from the tissues and entraps them into the circulation compartment. ${ }^{26}$ As free drugs diffuse into the lipid compartment, a concentration gradient is created to remove the drugs. Redistribution of amitriptyline to the tissues is also likely to occur. It is favorable that the toxicant-entrapped lipids redistribute to the liver for elimination. ${ }^{27}$ The large residence in the circulation and liver led to an ideal antidote of the anionic squarticles. The low amitriptyline release from the squarticles assured the ability to transfer the drug to the liver without leakage.

The infused antidotes could significantly alter the pharmacokinetics and biodistribution of amitriptyline. The reduced organ concentration and increased plasma concentration of the drug indicate the capability of anionic squarticles to restore organ function and eliminate amitriptyline. This supports the concept of the lipid sink. Although ILEs could sequester the drug from the organs, a major concern is that ILEs increase plasma triglycerides resulting in hypertriglyceridemia. ${ }^{7}$ This issue can be resolved by the treatment of anionic squarticles due to the absence of soybean oil in the formulations. On the other hand, the dosing of cationic squarticles did not significantly increase plasma amitriptyline concentration according to amitriptyline pharmacokinetics. This could be due to the preferable retention of the cationic squarticles in the peripheral organs, based on real-time and ex vivo imaging.

Successful scavenging of overdosed drugs should entail their inactivation rather than extraction. The organs of RES, such as liver and spleen, play a predominant role in clearing out the xenobiotic lipid particles. The macrophages in the liver and spleen are important for the uptake of ILEs for their metabolism as endogenous chylomicrons. ${ }^{24}$ In the rat study, squarticles and ILEs accelerated the transport of amitriptyline into the liver. The group administered saline showed no amitriptyline in the liver. This may lead to the high death rate of this group due to the negligible elimination of amitriptyline, although saline could ameliorate MAP reduction caused by the drug. The positive effect of saline on MAP could result from the dilution of amitriptyline in the circulation. This is confirmed by the low plasma level of amitriptyline in the group treated with saline. The smaller nanoparticles stay longer in the circulation before entering the RES. ${ }^{28}$ Liver clearance is relatively higher for positively charged nanoparticles than for negative ones. ${ }^{29}$ These factors result in greater delivery of cationic squarticles to the liver. The nanoparticles with small size $(<100 \mathrm{~nm})$ still end up in the liver since they can partition into the liver via fenestrated capillaries. ${ }^{1}$ The opsonization of nanoparticles is involved in both the liver and spleen. However, the uptake of squarticles by the spleen was low. The nanoparticulate size of $>200 \mathrm{~nm}$ is more likely cleared by the spleen because of the interendothelial cell slit of the spleen $(\sim 200 \mathrm{~nm}){ }^{30}$ The ex vivo bioimaging highlighted a greater retention of anionic squarticles in the spleen than the cationic ones. This could be due to the prolonged circulation allowing greater exposure to the spleen macrophages. ${ }^{31}$ However, this discrepancy between anionic and cationic nanosystems was not large after they were subjected to statistical analysis $(p>0.05)$.

Amitriptyline is quickly distributed from the blood into the highly perfused organs including the brain, heart, lungs, and liver. ${ }^{23}$ The side effects of TCAs on brain and heart correlate with the drug concentration in the organs. One principal finding in this report was the capability of squarticles to lower the amount of amitriptyline in the highly perfused organs. Although ILEs could decrease the drug concentration in the brain and lungs, soybean oil droplets easily expose to the heart; ${ }^{32}$ this can lead to increased cardiotoxicity. A large volume of ILE infusion also creates pulmonary complications such as adult respiratory distress syndrome and fat emboli. ${ }^{10,26}$ These effects may not be found in the case of squarticles. The cationic squarticles showed relatively higher transport to the brain compared to the anionic nanoparticles. The positively charged nanoparticles may facilely attach to the negatively charged clathrin-coated pits at the brain endothelial cells. ${ }^{33}$ Another observation was the higher accumulation of cationic squarticles in the lungs, compared to the anionic ones. A large particle size is imperative for the lung residence of lipid-based nanoparticles because of the high permeability of pulmonary alveoli. ${ }^{34}$ Squarticles effectively sequestered amitriptyline in the lungs where the drug 
concentration was very high. ILEs exhibited a comparable effect with the squarticles on reducing the concentration of amitriptyline in the lungs.

The association between amitriptyline and the lipid reservoir in blood offers the benefit of cardiac recovery. In addition to the lipid compartment effect, ILEs can improve the amitriptyline-induced cardiotoxicity through amelioration of calcium flux into the myocardium and fatty acid transport into cardiac mitochondria. ${ }^{35}$ This is the reason why ILEs restored MAP to near normal, although the fat emulsions did not significantly entrap amitriptyline from the heart. This improvement is mainly due to the free fatty acids in soybean oil. This is not the case for squarticles. Besides the strong affinity to lipophilic compounds, squalene can act as a vital antioxidant to exert cytoprotective effect. ${ }^{36}$ Previous studies ${ }^{14,37}$ have demonstrated that squalene is efficacious in stabilizing the mammalian cell membrane in cyclophosphamide- and isoproterenol-elicited cardiotoxicity. Even though the cationic squarticles extracted amitriptyline from the highly perfused organs and largely located it in the liver for elimination, this nanosystem did not accomplish the end point of MAP recovery and lethality reduction; this can be associated with the faster release of amitriptyline from the cationic squarticles than from the anionic ones after entrapment. Although the cationic squarticles extracted the drug for sequestration, these amitriptyline-loaded nanoparticles quickly redistributed to the tissues, inducing toxicity. Another possibility was the superiority of the cationic character for interacting with the negatively charged biomembrane to activate membrane damage. ${ }^{38}$ The positively charged nanoemulsions also caused increased hemolysis in the circulation, ${ }^{39}$ complicating the toxicity. Further study is necessary to elucidate the possible mechanisms related to the unsatisfactory outcome of cationic squarticles.

The anionic squarticles totally recovered MAP and improved the survival rate after administration of amitriptyline overdose. The lack of cationic surfactant in the formulations also reduced the possibility of nanotoxicity. Squalene possesses excellent biosafety with no sign of irritation and allergy. ${ }^{40}$ For instance, MF59 ${ }^{\circledR}$ containing squalene as the vaccine adjuvant is well documented to be safe without adverse effects after injection. ${ }^{41}$ The preliminary safety result of applying a bolus of $1.5 \mathrm{~mL} / \mathrm{kg}$ and the following $6 \mathrm{~mL} / \mathrm{kg} / \mathrm{h}$ infusion of anionic squarticles showed a constant MAP baseline and no rat death during a 120 min period.

\section{Conclusion}

TCA poisoning presents a principal concern in intensive care units. The acute character of the drug overdose requires the development of therapeutic intervention with high efficiency for reducing mortality. In this study, the treatment of amitriptyline intoxication was demonstrated using squarticles as the nanoantidotes. Squarticles are capable of sequestering amitriptyline owing to the potent affinity to, and entrapment of, the drug. The leakage of amitriptyline from squarticles was low after entrapment. Squarticles can extract amitriptyline from the highly perfused organs to the circulation, ameliorating the hypotension and survival rate caused by drug overdose. The findings from bioimaging and pharmacokinetics support the lipid sink as the major mechanism of nanodetoxification. The anionic squarticles revealed better detoxification compared to the cationic nanosystems because of the higher affinity to amitriptyline and greater efficiency in rerouting the drug back into the plasma. Our results suggest the potential of anionic squarticles for intoxication therapy. It would be advantageous to design a platform of nanoantidotes that can treat poisoning by a class of drugs. Further systematic study is required to evaluate the effect of squarticles on the intoxication of other TCAs.

\section{Acknowledgments}

The authors are grateful to the Ministry of Science and Technology of Taiwan (MOST-105-2320-B-182-010-MY3) and Chang Gung Memorial Hospital (CMRPD1F0231-3) for the financial support.

\section{Disclosure}

The authors report no conflicts of interest in this work.

\section{References}

1. Damitz R, Chauhan A. Parenteral emulsions and liposomes to treat drug overdose. Adv Drug Deliv Rev. 2015;90:12-23.

2. Rudd RA, Aleshire N, Zibbell JE, Gladden RM. Increases in drug and opioid overdose deaths - United States, 2000-2014. Morb Mortal Wkly Rep. 2016;64:1378-1382.

3. Hawton K, Bergen H, Simkin S, et al. Toxicity of antidepressants: rates of suicide relative to prescribing and non-fatal overdose. Br J Psychiat. 2010;196:354-358.

4. Aydin B, Hocaoglu N, Micili SC, Ergur BU, Kalkan S. Effects of 2-hydroxypropyl-beta-cyclodextrin on cardiovascular signs of amitriptyline poisoning in a rat model. Cardiovasc Toxicol. 2016;16: 374-380.

5. Agarwala R, Ahmed SZ, Wiegand TJ. Prolonged use of intravenous lipid emulsion in a severe tricyclic antidepressant overdose. $J$ Med Toxicol. 2014; 10:210-214.

6. Cao D, Heard K, Foran M, Koyfman A. Intravenous lipid emulsion in the emergency department: a systematic review of recent literature. J Emerg Med. 2015;48:387-397.

7. Harvey M, Cave G, Ong B. Intravenous lipid emulsion-augmented plasma exchange in a rabbit model of clomipramine toxicity: survival, but no sink. Clin Toxicol. 2014;52:13-19.

8. Wiedmer SK, Lokajová J. Capillary electromigration techniques for studying interactions between analytes and lipid dispersions. J Sep Sci. 2013;36:37-51. 
9. Levine M, Skolnik AB, Ruha AM, Bosak A, Menke N, Pizon AF. Complications following antidotal use of intravenous lipid emulsion therapy. J Med Toxicol. 2014;10:10-14.

10. Levine M, Brooks DE, Franken A, Graham R. Delayed-onset seizure and cardiac arrest after amitriptyline overdose, treated with intravenous lipid emulsion therapy. Pediatrics. 2012;130:e432-e438.

11. Graham LM, Nguyen TM, Lee SB. Nanodetoxification: emerging role of nanomaterials in drug intoxification treatment. Nanomedicine. 2011;6:921-928.

12. Aljuffali IA, Sung CT, Shen FM, Huang CT, Fang JY. Squarticles as a lipid nanocarrier for delivering diphencyprone and minoxidil to hair follicles and human dermal papilla cells. AAPS J. 2014;16:140-150.

13. Kim SK, Karadeniz F. Biological importance and applications of squalene and squalane. Adv Food Nutr Res. 2012;65:223-233.

14. Senthilkumar S, Devaki T, Manohar BM, Babu MS. Effect of squalene on cyclophosphamide-induced toxicity. Clin Chim Acta. 2006;364: 335-342.

15. Liu KS, Chen YW, Aljuffali IA, Chang CW, Wang JJ, Fang JY. Topically applied mesoridazine exhibits the strongest cutaneous analgesia and minimized skin disruption among tricyclic antidepressants: the skin absorption assessment. Eur J Pharm Biopharm. 2016;105:59-68.

16. Hsieh PW, Wen CJ, Yu HP, Aljuffali IA, Huang YH, Fang JY. Nanostructured lipid carriers containing a high percentage of a Pluronic copolymer increase the biodistribution of novel PDE4 inhibitors for the treatment of traumatic hemorrhage. J Biomed Nanotechnol. 2014;10: $1520-1535$.

17. Leroux JC. Injectable nanocarriers for biodetoxification. Nat Nanotechnol. 2007;2:679-684

18. Mahajan S, Mahajan RK. Interactions of phenothiazine drugs with surfactants: a detailed physicochemical overview. Adv Colloid Interf Sci. 2013;199-200:1-14.

19. Ernsting MJ, Murakami M, Roy A, Li SD. Factors controlling the pharmacokinetics, biodistribution and intratumoral penetration of nanoparticles. J Control Release. 2013;172:782-794.

20. French D, Smollin C, Ruan W, Drasner K. Wu A. Partition constant and volume of distribution as predictors of clinical efficacy of lipid rescue for toxicologic emergencies. Clin Toxicol. 2011;49:801-809.

21. Chen N, Huang Y, Wang Y. Bioinspired affinity DNA polymers on nanoparticles for drug sequestration and detoxification. Biomaterials. 2014;35:9709-9718.

22. Howell BA, Chauhan A. Interaction of cationic drugs with liposomes. Langmuir. 2009;25:12056-12065.

23. Litonius E, Niiya T, Neuvonen PJ, Rosenberg PH. No antidotal effect of intravenous lipid emulsion in experimental amitriptyline intoxification despite significant entrapment of amitriptyline. Basic Clin Pharmacol Toxicol. 2012;110:378-383.

24. Heinonen JA, Litonius E, Backman JT, Neuvonen PJ, Rosenberg PH. Intravenous lipid emulsion entraps amitriptyline into plasma and can lower its brain concentration - an experimental intoxication study in pigs. Basic Clin Pharmacol Toxicol. 2013;113:193-200.

25. Harvey M, Cave G. Case report: successful lipid resuscitation in multidrug overdose with predominant tricyclic antidepressant toxidrome. Int J Emerg Med. 2012;5:8.
26. Cevik SE, Tasyurek T, Guneysel O. Intralipid emulsion treatment as an antidote in lipophilic drug intoxications. Am J Emerg Med. 2014;32: 1103-1108.

27. Engels PT, Davidow JS. Intravenous fat emulsion to reverse hemodynamic instability from intentional amitriptyline overdose. Resuscitation. 2010;81:1037-1039.

28. Hörmann K, Zimmer A. Drug delivery and drug targeting with parenteral lipid nanoemulsions - a review. J Control Release. 2016;223: 85-98

29. Keck CM, Jansch M, Müller RH. Protein adsorption patterns and analysis on IV nanoemulsions - the key factor determining the organ distribution. Pharmaceutics. 2012;5:36-68.

30. Almeida JPM, Chen AL, Foster A, Drezek R. In vivo biodistribution of nanoparticles. Nanomedicine. 2011;6:815-835.

31. Hsu CY, Chen CH, Aljuffali IA, Dai YS, Fang JY. Nanovesicle delivery to the liver via RBP and PDGF receptors: how the targeting ligands affect biodistribution. Nanomedicine. 2017;12:317-331.

32. Perichon D, Turfus S, Gerostamoulos D, Graudins A. An assessment of the in vivo effects of intravenous lipid emulsion on blood drug concentration and haemodynamics following oro-gastric amitriptyline overdose. Clin Toxicol. 2013;51:208-215.

33. Voigt N, Henrich-Noack P, Kockentiedt S, Hintz W, Tomas J, Sabel BA. Surfactants, not size or zeta-potential influence blood-brain barrier passage of polymeric nanoparticles. Eur J Pharm Biopharm. 2014;87: 19-29.

34. Beloqui A, Solinís MA, Delgado A, Évora C, del Pozo-RodríguezGascón A. Biodistribution of nanostructured lipid carriers (NLCs) after intravenous administration to rats: influence of technological factors. Eur J Pharm Biopharm. 2013;84:309-314.

35. Bania TC, Chu J, Perez E, Su M, Hahn IH. Hemodynamic effects of intravenous fat emulsion in an animal model of severe verapamil toxicity resuscitated with atropine, calcium, and saline. Acad Emerg Med. 2007; 14:105-111.

36. Huang ZR, Lin YK, Fang JY. Biological and pharmacological activities of squalene and related compounds: potential uses in cosmetic dermatology. Molecules. 2009;14:540-554.

37. Sabeena Farvin KH, Anandan R, Hari Senthil Kumar S, Shiny KS, Sankar TV, Thankappan TK. Effect of squalene on tissue defense system in isoproterenol-induced myocardial infarction in rats. Pharm Res. 2004;50:231-236.

38. Fröhlich E. The role of surface charge in cellular uptake and cytotoxicity of medical nanoparticles. Int J Nanomed. 2012;7:5577-5591.

39. Bruxel F, Cojean S, Bochot A, et al. Cationic nanoemulsion as a delivery system for oligonucleotides targeting malarial topoisomerase II. Int $J$ Pharm. 2011;416:402-409.

40. Fox CB. Squalene emulsions for parenteral vaccine and drug delivery. Molecules. 2009;14:3286-3312.

41. Schultze V, D’Agosto V, Wack A, Novicki D, Zorn J, Hennig R. Safety of MF59 adjuvant. Vaccine. 2008;26:3209-3222.
International Journal of Nanomedicine

\section{Publish your work in this journal}

The International Journal of Nanomedicine is an international, peerreviewed journal focusing on the application of nanotechnology in diagnostics, therapeutics, and drug delivery systems throughout the biomedical field. This journal is indexed on PubMed Central, MedLine, CAS, SciSearch $®$, Current Contents $\AA /$ Clinical Medicine,

\section{Dovepress}

Journal Citation Reports/Science Edition, EMBase, Scopus and the Elsevier Bibliographic databases. The manuscript management system is completely online and includes a very quick and fair peer-review system, which is all easy to use. Visit http://www.dovepress.com/ testimonials.php to read real quotes from published authors. 